\title{
Acute pain syndromes in cancer
}

Volume 4 Issue 3 - 2016

\section{Introduction}

Cancer management is a multidimensional and requires an interdisciplinary approach. It requires a close interaction between various specialties like medical, radiation, surgical oncology, anesthesiology and pain and palliative care services in addition to interventional physician. The various pharmacological (like chemotherapy) and non-pharmacological (surgical, radiation) intervention for diagnostic and therapeutic purposes have associated adverse effects and pain is one of them. Pain is a complex phenomenon and remains to be one of the feared symptoms in cancer patient leading to poor quality of life. The prevalence of pain in cancer patients varies from $52 \%$ to $77 \%{ }^{1,2}$ The pain may be of nociceptive, neuropathic or mixed origin in these patients. ${ }^{2}$ The complexities and high prevalence of pain in cancer patients have led to origin of "acute pain syndromes in cancer" terminology. It comprises various etiologies responsible for pain in cancer patients. These may be of acute onset and if not managed timely may lead to chronic pain. Also, on occasions, there may be acute exacerbations in the background of chronic pain or there may have transitory breakthrough pains. ${ }^{3}$

\section{Acute pain syndromes in cancer}

Cancer requires appropriate assessment (diagnostic interventions), treatment interventions (hormonal therapy, chemotherapy, radiotherapy, surgery) or palliative symptom management. ${ }^{1,3}$ These all interventions may lead to acute pain syndrome including neuropathies due to injuries to nerves during surgical intervention, acute neurotoxicity due to chemotherapeutic agents and acute radiation induced injuries (Table 1). The severity of these pain syndromes depend on the site and extent of the tumor mass. The prevention and timely management of these acute pain syndromes is essential as any sort of acute pain in cancer patients induces neuronal remodelling and sensitization which finally may translate in chronic pain leading to poor quality of life. ${ }^{4}$ In fact, acute pain should be red flag for ongoing tissue injury leading to nociceptive and neural compromise., These syndromes have a complex outcome and involve a complex conglomeration of physiological, emotional, cognitive, social, and environmental factors.

Understanding of cancer pain syndromes is essential to understand etiology causing pain, guides for appropriate assessment, helps in planning treatment management and assist in patient counseling. ${ }^{3}$ This mandates all physicians involved in cancer management to understand these pain syndromes for optimal management.

Etiology of acute pain syndromes: The acute pain syndromes may have various etiologies. Broadly, these syndromes may be categorized based on character of pain, nature of therapy leading to pain syndrome or specific tissue involvements leading to pain syndrome.

Acute pain syndromes based on character of pain: Tumor-related nociceptive pain syndromes: $:^{1,5,6}$ The tumor can invade various somatic or visceral structures like bone, joint, muscle, or connective tissue leading to acute pain. Bone pain syndrome is one of the commoner syndrome in cancer patients. The vertebrae involvement is common

\section{Rakesh Garg}

Assistant Professor, Department of Onco-Anesthesia and Palliative Medicine, India

\section{Correspondence: Rakesh Garg, Department of} Anaesthesiology, Pain and Palliative Care, Dr BRAIRCH, Room No.139, Ist floor, All India Institute of Medical Sciences, Ansari Nagar, New Delhi- I 10029, India, Tel +9| 9810394950; +9 I 9868398335,Email drrgarg@hotmail.com

Received: December 07, 2015 | Published: February 05, 2016

and may have bony pain. However bony involvement without pain may also be observed in cancer patients. This bony involvement may at times lead to neural compromise and manifests neuropathic component of pain as well. Thus is essential for timely recognition of this syndrome and appropriate management. The visceral organs and their adjoining connective tissue may also be involved leading to obstruction, infiltration, or compression of these viscera and manifesting as visceral nociceptive pain syndromes. Diagnosis could may be made by appropriate assessment. However, at times, pain manifestation precedes diagnosis of cancer. Such pain syndromes may occur or get exacerbated due to cancer treatment modalities.

Tumor-related neuropathic pain syndromes $[3,4]$ : The neuropathic pain syndromes in cancer patient may occur due to tumor infiltration or compression of nerve, plexus, or roots or due to tumor related products causing neural damage. These may also occur due to treatment related neural toxicity.

Acute pain syndromes related to cancer treatment: The treatment for cancer requires a multidisciplinary approach including chemotherapy, radiation therapy and surgical interventions. These interventions may lead to acute pain syndromes in cancer patient due to their side efefcts profile. Though the nociceptive pain is common but neuropathic pain is more commoner with treatment related acute pain syndromes in cancer patients. It is essential to understand thee syndromes to differentiate form pain due to tumor recurrence or tumor metastasis.

Surgery associated pain syndromes: Acute postsurgical pain commonly exists after the cancer surgery and severity is based on extent and site of surgical intervention. ${ }^{7}$ The tissue damage leads to nociceptive pain and nerve injury leads to neuropathic component of pain. The postsurgical pain syndromes have been described for various specific interventions. These includes post-mastectomy, post- 
amputation, post-thoractomy, and other surgical pain states. ${ }^{5,8}$ These pain syndromes precede with some acute insult and inadequate pain management in the initial stages.

Table I Acute Pain Syndromes in Patients with Cancer ${ }^{6}$

Acute pain associated with diagnostic procedures

Pain due to lumbar puncture for CSF analysis

Bone-marrow biopsy for analysis

Peripheral or central catheter insertion

Paracentesis

Thoracentesis

Acute pain associated with analgesic techniques

Spinal opioid hyperalgesia syndrome

Acute pain following Sr-89 therapy of metastatic bone pain

Acute Surgical pain

Acute pain associated with other therapeutic procedures

Pleurodesis

Tumor embolization

Nephrostomy insertion

Bone marrow transplantation and associated immunosupresion leading to mucositis

Acute pain associated with chemotherapy

Pain from intravenous or intra-arterial infusion

Intraperitoneal chemotherapy

Intrathecal chemotherapy

Mucositis

Neuropathy

Bone or muscle pain

5-fluorouracil-induced angina

Acute pain associated with hormonal therapy

Painful gynecomastia

Luteinizing hormone releasing factor tumor flare in prostate cancer

Hormone-induced acute pain flare in breast cancer

Acute pain associated with immunotherapy

Arthralgia and myalgia from interferon and interleukin

Acute pain associated with radiation therapy

Mucositis

Enteritis and proctocolitis

Brachial plexopathy

Acute tumor-related pain

Vertebral collapse and other pathological fractures

Acute obstruction of hollow viscus

Intracranial hypertension

Hemorrhage into tumor

Acute pain associated with infection

Myalgia and arthralgias

Wounds or abscesses

a. Post-thoracotomy pain occurs after thoractomies for cancer surgery and occurs due to surgical interruption or injury to the intercoastal nerves. ${ }^{5}$ Traction on the ribs and rib resection for surgical exposure leads to such damage. Patient presents with immediate postoperative pain and is characterized by an aching sensation in the distribution of the incision and sensory loss, with or without autonomic changes.

b. Post-mastectomy pain occurs after the surgical procedure on the breast especially when associated with after axillary and lymph node dissection. ${ }^{5}$ The pain occurs due to surgical damage of the intercostal brachial nerve. This pain may have acute onset or in some cases presents late upto 6 months after surgical intervention. Patient presents with tight, constricting, burning pain in the posterior aspect of the arm and axilla radiating across the anterior chest wall which increases by movement of the arm and relieved by its immobilization.

c. Post-radical neck surgery pain syndrome presents usually late but in some cases may have acute presentation. ${ }^{5}$ Patient presents with burning dysesthesia and shock-like shooting and lancinating pains.

d. Post-amputation pain syndrome is usually a chronic phenomenon. However various surgical causes leads to acute pain manifestation and if not managed adequately, it has high propensity for chronic manifestation. ${ }^{5}$ The presentation of pain intensity, frequency, and duration, as well as provocative and palliative factors, differ among patients.

Chemotherapy and hormonal therapy associated pain syndromes: Chemotherapy drugs have associated adverse effects and many of them are self limiting. The acute toxicity may manifests as pain and requires aggressive and timely intervention. Treatment having steroid as part of the chemotherapeutic regimen may lead to bone necrosis leading to somatic bony pain. The use of intraperitoneal chemotherapy may cause acute abdominal pain in addition to other toxic effects on abdominal body organs. The chemotherapeutic drugs like vincristine, platinum, taxanes, thalidomide, bortezimib are prone for causing painful dysesthesias. ${ }^{8,9,10}$ These agents produce a symmetric polyneuropathy as a result of a subacute chronic axonopathy. ${ }^{5}$ Paclitaxel-associated acute pain syndrome is well described and occurs within days after each dose. ${ }^{11,12}$ It is used for breast, ovarian, lung, and head and neck cancers. The pain steadily increases in severity with increasing paclitaxel total doses. Pain may be localized or generalized variably. Patients with worse paclitaxel-associated acute pain syndrome severities may finally presents with features of chemotherapy- induced peripheral neuropathy. ${ }^{13}$ It is characterized first by paresthesias, followed by dysesthesias. Pain may be localized to the large axial muscles in the shoulders, paraspinal regions, pelvis, and thighs. ${ }^{13}$ The hormonal therapies are also associated with pain like arthralgias occurring after aromatase inhibitors. ${ }^{10}$

Radiation therapy associated pain syndromes: ${ }^{1,5,8}$ Many tumors requires radiation therapy as radical treatment or an adjunct to other management including chemotherapy and surgical intervention. The radiation exposure causes various bodily changes leading to acute manifestation of pain. Radiation may lead to osteonoecrosis of bone and lead to bony pain. The tissue toxicity due to radiation exposure depends not only the dose of radiation but also the site of radiation exposure. The abdominal radiation therapy may present as acute abdominal manifestation and pain may be presenting complaint in many of these patients. The most common radiation-induced neural insult for breast cancers is radiation-induced brachial plexopathy but manifests late. The pathophysiology of radiation-induced neuropathies is not clear but may be related to radiation-induced fibrosis and associated factors such as ischemia, oxidative stress and inflammation as well as other pathophysiologic mechanisms suspected of contributing to radiation-induced neuropathies.

\section{Acute pain syndromes related to tumor invasion}

Bone invasion by tumor: The bony involvement may be primary, metastatic or locally advanced. The bony pain due to neoplasm may be due to marrow infiltration leading to activation of mechanoreceptive nociceptors, invasion of the periosteum, lytic lesion causing fractures, release of chemical mediators that activate nociceptors. ${ }^{5}$ The bony involvement and its destruction may have associated neurological 
compromise like for vertebras or nerve compression of other skeletal bones. The movement of these bones may further exacerbate acute pain.

Tissue invasion by tumor: The tumor may infiltrate various body tissues having somatic or neuropathic component. This may be due to infiltration, compression, traction or vascular compromise. ${ }^{3,5}$

Management of acute pain syndromes in cancer: The management for acute pain syndromes in cancer patients requires a holistic and multidisciplinary approach including pharmacological, interventional, physical, psychological, rehabilitative, and alternative modalities. ${ }^{1,5,12}$ This is essential as these syndromes have the propensity to continue as chronic pain and becomes difficult to treat. So, acute pain syndromes of cancer requires aggressive management at its initial presentation. The pain management needs to be individualized based on etiology of acute injury leading to pain syndrome. At times, an intervention targeted at the etiology of pain needs major attention to alleviate pain faster and satisfactorily.

Pharmacological approaches: The opioid and non-opioid drugs along with appropriate use of adjuvants provides optimal pain control in acute pain syndromes of cancer. ${ }^{3,9}$

Opioids [3]: The acute pain syndrome may have more severity and requires appropriate drug selection and opioids remains the mainstay of treatment. The "analgesic ladder" approach of WHO is the conventional method for drug selection.

Non-opioid and adjuvant analgesics $[3,14,15]$ : These include drugs like acetaminophen and the non-steroidal anti-inflammatory drugs (NSAIDs). The adjuvants includes gabapanternoids, anticonvulsants, serotonin-norepinephrine reuptake inhibitor (SNRI), steroids, local anesthetics, N-methyl-D-aspartate receptor antagonists etc. The adjuvants primarily decreases the opioid requirements and have more effective role in prevention of chronicity of pain. Bony pain requires use of adjuvants like bisphosphonates, radiopharmaceutical drugs, and calcitonin. The pain associated with malignant bowel obstruction requires anticholinergic drugs, octreotide, and corticosteroids. ${ }^{10}$

Regional analgesia [15]: The use of regional and central neuraxial blocks have paramount role in acute pain syndrome specially after surgery associated acute pain syndromes. The use of local anaesthetics along with adjuvants like opioids and alphs-2-agonist have been found to provide optimal pain control.

Prevention of persistent postsurgical Pain: It is essential to provide optimal control of the pain specially for surgical intervention to avoid phenomenon of persistent postsurgical pain..$^{10,15,16}$ The incidence of chronic post surgical pain varies with surgical interventions. ${ }^{10,17}$ Though the exact mechanisms is not known. Factors which has propensity for chronic pain postoperatively includes: ${ }^{8}$

a. Preoperative factors: previous pain duration prior to surgery, previous history of surgery like recurrence; psychological

b. Intraoperative factors: extent of surgical intervention, the site of surgery, the technique of surgical insult and involvement of the nerve.

c. Postoperative factors: inadequate control of acute pain, associated concomitant radio or chemo-therapy, anxiety.

\section{Conclusion}

The cancer management requires holistic and multidisciplinary approach. The cancer management required various intervention for diagnosis, cure or palliative management. These may lead to occurrence of pain. These requires aggressive management using a multimodal approach. This would not only improve patient satisfaction and quality of life but also prevent occurrence of chronic pain syndromes.

\section{References}

1. Everdingen MHJ, Rijke JM, Schouten HC, et al. Prevalence of pain in patients with cancer: a systematic review of the past 40 years. Ann Oncol. 2007;18(9):1437-1449.

2. Martin LA, Hagen NA. Neuropathic pain in cancer patients: mechanisms, syndromes and clinical controversies. J Pain Sym Manage. 1997;14(2):99-107.

3. Portenoy RK, Lesage P. Management of cancer pain. Lancet 353(9165): 1695-1700.

4. Carr DB, Goudas LC (1999) Acute Pain. Lancet. 1999;353:2051-2058.

5. Nersesyan H, Slavin KV. Current aproach to cancer pain management Availability and implications of different treatment options. Ther Clin Risk Manag. 2007;3(3):381-400.

6. Portenoy RK. Pain syndromes in patients with cancer and HIV/AIDS In: Portenoy RK (ed.), Contemporary diagnosis and management of pain in oncologic and AIDS patients. Handbooks on Healthcare, Newton, Pennsylvania, USA, 1998. p. 44-70.

7. Amaya F, Hosokawa T, Okamoto A, et al. Can Acute Pain Treatment Reduce Postsurgical Comorbidity after Breast Cancer Surgery? A Literature Review. Biomed Research International. 2005;1:1-8.

8. Burton AW, Fanciullo GJ, Beasley RD, et al. Chronic pain in cancer survivor: a new frontier. Pain Medicine. 2007;8(2):189-198.

9. Fernandes R, Mazzarello S, Majeed H, et al. Treatment of taxane acute pain syndrome (TAPS) in cancer patients receiving taxane-based chemotherapy-a systematic review. Support Care Cancer. 2015.

10. Goudas LC, Bloch R, Gialeli-Goudas M, et al. The epidemiology of cancer pain. Cancer Invest. 2005;23(2):182-190.

11. Reeves BN, Dakhil SR, Slaon JA, et al. Further Data Supporting That Paclitaxel-Associated Acute Pain Syndrome Is Associated With Development of Peripheral Neuropathy. Cancer. 2012;118(20):5171-5178.

12. Loprinzi CL, Reeves BN, Dakhil SR, et al. Natural History of PaclitaxelAssociated Acute Pain Syndrome: Prospective Cohort Study NCCTG N08C1. J Clin Oncol. 2011;29(11):1472-1478.

13. Loprinzi CL, Kami M, Wolf SL, et al. The Paclitaxel Acute Pain Syndrome: Sensitization of Nociceptors as the Putative Mechanism. Cancer J.2007; 13(6):399-403.

14. Lussier D, Huskey AG, Portney RK. Adjuvant analgesics in cancer pain management. The Oncologist. 2004;9(5):571-591.

15. Mercadante S, Arcuri E. Breakthrough pain in cancer patients: pathophysiology and teratment. Cancer Treat Rev. 1998;24(6):425-432.

16. MacDonald L, Bruce J, Scott NW, et al. Long-term follow-up of breast cancer survivors with post-mastectomy pain syndrome. $\mathrm{Br} J$ Cancer. 2005; 92(2):225-230.

17. Macrae WA. Chronic post-surgical pain: 10 years on. Br J Anaesth. 2008;101(1):77-86. 